\title{
The Prescription Drug Epidemic in Appalachian Elderly
}

Suzanne Holroyd, $\mathrm{MD}^{1}$

\section{Author Affiliations:}

1. Department of Psychiatry and Behavioral Medicine, Marshall University, Huntington, West Virginia

The author has no financial disclosures to declare and no conflicts of interest to report.

\section{Corresponding Author:}

Suzanne Holroyd, MD

Department of Psychiatry and Behavioral Medicine

Marshall University Joan C. Edwards School of Medicine

Huntington, West Virginia

Email: holroyds @marshall.edu 
There is another drug epidemic in Appalachia but it's not what you think. While the opioid and heroin addiction crisis has rightfully come to the forefront of national awareness, there is another group of Americans being devastated by a prescription drug epidemic and it is barely being noticed. What is this other silent epidemic which is also causing death and disability, creating immense caregiver stress in families and sending health care costs skyrocketing upward? It is the over prescribing of drugs to the elderly population in Appalachia. Overprescribing often causes confusion, misdiagnosed as dementia, and results in inappropriate nursing home placement, falls and associated physical morbidity.

Appalachia is at particular risk for such an epidemic, given the especially high proportion of elderly in this region. For example, West Virginia has the second highest mean age of any state in the US, with one in six residents being over age 65 . By 2030, one in every four residents in WV will be over age 65 and the number who are 85 and over will double over the next two decades.

Given the large numbers of seniors, any increase of the proportion with physical or cognitive impairments creates huge emotional and financial burdens for families, communities and governmental agencies. Such seniors require caregivers, either family or paid, to maintain living in the home, or require placement in nursing homes. The stress of caregiving puts huge burdens on families, causing stress, depression, economic hardship and increased potential for abuse.

But what if many of these physical and cognitive impairments are just due to side effects of prescription drugs? Per the CDC, West Virginia has the highest rate of sedative/benzodiazepine use in the country and no other state is even a close second. As well, West Virginia is second in the nation for opioid prescriptions. Such medications are well known to cause sedation, falls and confusion in the elderly.

When a younger adult develops confusion or has a change in behavior, it is recognized as secondary to an underlying process or a medication side effect. However, when an elderly person becomes confused or behaves strangely, it is too often accepted as part of the natural aging process. Having practiced in West Virginia for only two years, I have been unpleasantly surprised by the number of elderly misdiagnosed with "dementia" who, by simply reducing their medication (typically benzodiazepines, narcotics, muscle relaxants and hypnotics), suddenly no longer have "dementia”. And this isn't just the 80-90 year olds. I have also been referred patients in their 50s and 60s diagnosed with dementia who were simply delirious on their prescriptions. Notably, these are prescriptions that are being taken correctly as prescribed. Although exact statistics are currently lacking regarding the specific prescribing practices to the elderly in our region, given West Virginia has the highest prescribing of such medications and one of the oldest populations, I think it is easy to understand our elderly are receiving too many medications and that they are being harmed by them.

If there was a story in the news about a doctor who prescribed so many meds to a young adult that the adult became disabled and required 24 hour care, the public would rightly be outraged, recognize the prescribing as wrong and demand such prescribing be corrected. The same should hold true for our cherished elderly citizens.

My hope is for all practitioners to carefully examine the medication regimen of their elderly patients and stop narcotics, appropriately wean and taper benzodiazepines, and minimize all 
other medications. Many medications may have been useful earlier in life but now just add to confusion, memory problems, unsteady gait and other side effects. For example, anticholinergic medication may be prescribed for urinary incontinence and might have been useful in earlier years, but subsequently is no longer indicated and should be discontinued. More education regarding geriatric prescribing should also be helpful, as well as the development of more geriatric training programs in the state. The financial cost savings of having fewer disabled and confused elderly, not to mention the cost savings on medications that no longer would be prescribed, would be huge. Such a practice will help to bring about an end to this "other" Appalachian drug crisis. 